\title{
APLICACIONES DE CAUCHO RECICLADO: UNA REVISIÓN DE LA LITERATURA
}

\section{APPLICATIONS OF RECYCLED RUBBER: A LITERATURE REVIEW}

\author{
Gabriel Jaime Peláez Arroyave* \\ Sandra Milena Velásquez Restrepo" \\ Diego Hernán Giraldo Vásquez ${ }^{* *}$ \\ Fecha de recepción: 8 de agosto de 2016 \\ Fecha de revisión: 28 de septiembre de 2016 \\ Fecha de aprobación: 14 de febrero de 2017
}

Cómo citar: G. J. Peláez Arroyave, S. M. Velásquez Restrepo y D. H. Giraldo Vásquez, “Aplicaciones de caucho reciclado: una revisión de la literatura," Ciencia e ingeniería Neogranadina, vol. 27, no. 2, pp. 27-50. DOI: http:// dx.doi.org/10.18359/rcin. 2143

\section{RESUMEN}

La generación de residuos de caucho se ha convertido en una preocupación global por su impacto negativo en el medio ambiente y en la salud humana. La legislación ambiental sobre la disposición de este tipo de residuos ha venido aumentando las exigencias a los fabricantes, comercializadores y usuarios, con lo cual se ha acelerado la búsqueda de alternativas para el reaprovechamiento de los residuos de caucho. Este artículo presenta una revisión bibliográfica acerca de las principales tendencias en la utilización del caucho reciclado, incluyendo

Ingeniero de materiales; magíster en Ciencia e Ingeniería de Materiales. Se desempeña como investigador del Centro de Diseño y Manufactura del Cuero del Servicio Nacional de Aprendizaje (SENA). Miembro del grupo, Biomecánica, Materiales, TIC, Diseño y Calidad para el Sector cuero, plástico, caucho y sus cadenas productivas (Biomatic). Itagüí, Colombia. Correo electrónico:gajapear@gmail.com. ORCID: http://orcid.org/0000-0002-9540-9241

** Bioingeniera; especialista en Gerencia; magíster en Ingeniería. Se desempeña como líder de Innovación y Desarrollo Tecnológico del Centro de Diseño y Manufactura del Cuero del Servicio Nacional de Aprendizaje (SENA). Directora del grupo Biomecánica, Materiales, TIC, Diseño y Calidad para el Sector cuero, plástico, caucho y sus cadenas productivas (Biomatic). Itagüí, Colombia. Correo electrónico: smvelasquez@sena.edu.co. ORCID: http://orcid.org/0000-0002-6697-2801

*** Ingeniero Mecánico; magíster en Ingeniería. Se desempeña como profesor del Programa de Ingeniería de Materiales, de la Universidad de Antioquia. Director del grupo de Materiales Poliméricos, Medellín, Colombia. Correo electrónico: dhernan.giraldo@udea.edu.co. ORCID: http://orcid.org/0000-0002-4306-9978 
aplicaciones actualmente comercializadas y otras derivadas, tanto de estudios terminados, como de líneas de investigación en desarrollo. Las aplicaciones con mayor potencial de volumen consumido son del sector infraestructura y construcciones civiles, especialmente asfaltos, concretos y materiales aislantes para construcción liviana. Algunas aplicaciones con potencial en el futuro cercano son los filtros para la limpieza de aguas contaminadas y como componente en materiales compuestos con matriz termoplástica o en poliuretano. Por último, se evidencia la pertinencia de continuar investigando sobre esta temática que posee unos retos científicos de alta relevancia, los cuales ameritan la mayor atención por el problema ambiental significativo asociado al manejo de los residuos de caucho.

Palabras clave: Caucho reciclado, residuos de caucho, Ilantas fuera de uso, impacto ambiental, sostenibilidad.

\section{ABSTRACT}

The generation of rubber waste has become a global concern for its negative impact on the environment and human health. Environmental legislation on the disposal of this kind of residue has been increasing the demands on the manufacturers, traders, and users; therefore, has accelerated the search for alternatives to reclaim rubber waste. This article presents a bibliographical review of the main tendencies in the use of recycled rubber, including currently commercialized applications and others derived from finished studies or ongoing research lines. The applications with greater potential regarding consumed volume are related to infrastructure and civil construction sector, especially asphalts, concretes and isolators for lightweight construction. Some other applications with potential shortly are filters for cleaning contaminated water and as a component in thermoplastic matrix or polyurethane composites materials. Finally, it is evident the pertinence of continuing research on this subject that has scientific challenges of high relevance, which warrant the greatest attention for the management of rubber waste.

Keywords: Recycled rubber, rubber waste, tires out of use, environmental impact, sustainability.

\section{INTRODUCCIÓN}

Los materiales elastoméricos, conocidos como cauchos en la mayoría de países hispanoamericanos o como hules en otros, presentan una elasticidad y resistencia química que los hace insustituibles para llantas, calzado, tuberías, correas de transmisión o piezas para sellamiento de fluidos, por citar algunos productos para los que más se emplea el caucho como materia prima [1]. En 2014, el último año del cual se cuenta con estadísticas consolidadas sobre el mercado del caucho, el consumo mundial de esta materia prima fue de 28,9 millones de toneladas [2]. Se estima que en 2015 la demanda mun- 
dial de caucho aumentó un $0,7 \%$ respecto a 2014 y que entre 2016 y 2024 el consumo aumentará un 3,1\% anualmente en promedio [3], lo que confirma la tendencia creciente del mercado observada en las últimas décadas [4]. Desafortunadamente, las cifras sobre tendencias del mercado del caucho son reportadas esporádicamente por diferentes fuentes de información, de tal manera que al momento no es posible contrastar información y efectuar una evaluación cuantitativa consolidada sobre las tendencias del mercado de este tipo de materiales.

Para comprender los retos asociados al reciclaje de caucho, es pertinente tener en cuenta que los artículos en este material se fabrican mezclando elastómeros con aditivos orgánicos de bajo peso molecular, agentes vulcanizantes y cargas minerales con forma de partículas finas. La mezcla en estado crudo es sometida a un proceso de vulcanización en el cual se desarrolla una reacción química que forma una estructura entrecruzada altamente elástica; a este tipo de material se le denomina caucho virgen, para diferenciarlo del caucho reciclado. La reacción de vulcanización es térmicamente irreversible, por lo cual el reciclaje de los cauchos debe realizarse por trituración mecánica, métodos químicos o recuperación energética [1]. Esto implica grandes retos tecnológicos y logísticos para alcanzar tasas de recuperación de residuos compatibles con las necesidades actuales de mitigación de impacto ambiental.
Considerando que entre el 65 y el $70 \%$ del caucho producido en el mundo es utilizado para la fabricación de llantas [5], [6], debe tenerse en cuenta que cada año se generan aproximadamente 17 millones de toneladas de llantas fuera de uso (LLFU). Dado que el total de caucho en una llanta corresponde más o menos el 41 y el $55 \%$ de su peso, se tiene que entre 7 y 9 millones de toneladas de caucho provenientes de LLFU se disponen cada año a nivel mundial [7]. Para tener una idea del rápido crecimiento de esta problemática en un país en vía de desarrollo como Colombia, es pertinente indicar que en 2010 se dispusieron 42 mil toneladas de caucho proveniente de LLFU [8], mientras que en 2015 se generaron alrededor de 100 mil toneladas [9]. Se trata, entonces, de uno de los principales problemas asociados a la gestión de desechos sólidos en la actualidad, debido al efecto negativo directo que pueden traer sobre la salud humana y el medio ambiente, ya que contaminan el suelo, el agua y el aire. Las LLFU no son biodegradables y pueden constituir un ambiente favorable para reproducir plagas, ratas, mosquitos, etc. [10]; además de ser una potencial amenaza de incendio con generación de humareda tóxica que puede arder durante meses [11]. La Fig. 1 muestra la tendencia de aumento de la demanda mundial de caucho para la fabricación de llantas y la de generación de desperdicios de caucho proveniente de LLFU en la Unión Europea (UE). 


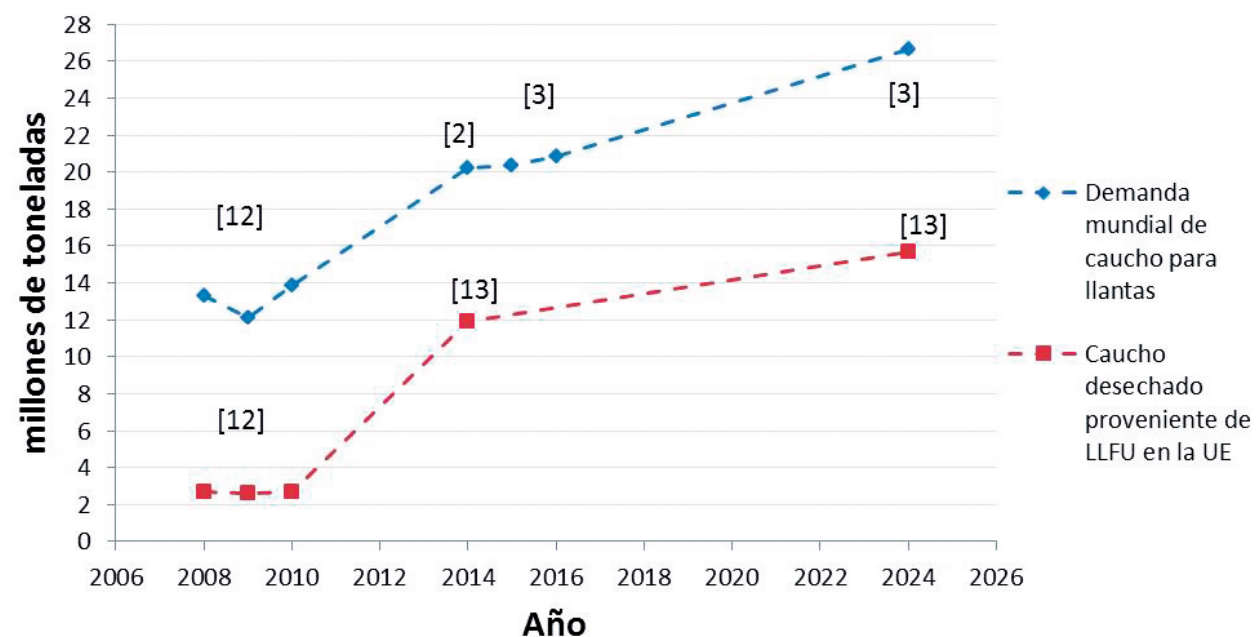

Fig. 1. Tendencia de la demanda mundial de caucho para la fabricación de llantas y de generación de desperdicios de caucho proveniente de LLFU en la UE

* La unión de los puntos es meramente ilustrativa.

Fuente: elaboración propia a partir de información de [2], [3], [12] y [13].

La problemática asociada a la disposición del caucho posconsumo y posproceso hizo que los gobiernos comenzaran a implementar de manera cada vez más decidida la formulación e implementación de regulaciones orientadas a disminuir la contaminación generada por este tipo de residuos. Es de destacar que algunas de esas regulaciones incentivan a la industria para que utilice caucho reciclado en sus procesos, tendencia en la cual Taiwán fue pionero en la implementación de dicha normatividad. Posteriormente, Estados Unidos, Japón y los países de la UE incursionaron en este campo, de manera que a hoy son las regiones con el mayor grado de desarrollo en cuanto a legislación sobre esta temática. Para tener una idea de la manera como ha evolucionado la normatividad en países como los de la UE, vale la pena reportar que en 1999 se impuso la directiva $1000 / 31 / E C$, que prohíbe la disposición de LLFU enteras o trituradas en los rellenos sanitarios; en 2000 apareció la directriz 2000/53/EC, que estableció que las llantas de los autos deben ser retiradas antes de que estos sean chatarrizados, y en 2009 la regulación 1222/2009 estipuló el etiquetado y seguimiento de las llantas [14], [15]. Se evidencia entonces la pertinencia de actualizar las políticas sobre la reutilización del caucho según las dinámicas del mercado, teniendo siempre como objetivo disminuir el negativo impacto sobre el medio ambiente, si no se hace una disposición adecuada de este tipo de materiales cuando terminan su vida útil primaria.

Por otro lado, se ha demostrado que el grado de desarrollo de la industria de reciclaje del caucho en cada país está directamente ligado a la legislación vigente [16], por lo cual se espera que, como la normatividad 
sobre residuos es cada vez más exigente, sea más necesario encontrar alternativas para el uso de caucho reciclado. No basta con fomentar la aplicación de las normas establecidas para la industria del caucho y sus usuarios, también es importante buscar aplicaciones en las cuales se puedan emplear los residuos generados.

Las regulaciones para tratar de atenuar el efecto negativo ambiental generado por los residuos de LLFU pueden ser clasificadas en los tres modelos que se describen en la Tabla 1, en la cual también se indican países que las han adoptado y el año cuando estas se implementaron [14], [15], [17]-[21].

Dado que en los países desarrollados las regulaciones sobre la disposición de estos desechos han venido tornándose más estrictas con el paso de los años [8], [16], la globalización ha llevado a que países en vías de desarrollo también adopten políticas ambientales cada vez más exigentes, bien sea por consciencia ambiental o por exigencias de los tratados de comercio que establecen con países desarrollados, o una combinación de ambos factores. Para continuar con el caso de Colombia, hay que anotar que en el país se implementó en 2010 la Resolución 1457, siguiendo el modelo de Responsabilidad Extendida del Productor (REP) para la gestión de LLFU [22], y así se delegó a los productores e importadores que manejan más de 200 llantas al año la responsabilidad de implementar los sistemas de recolección y gestión ambiental específicos para esta clase de desechos. En esa normatividad se establece que a partir de 2012 debía recolectarse y gestionarse el $20 \%$ de las llantas comercializadas en el país, con incrementos anuales del $5 \%$ hasta alcanzar un mínimo del $65 \%$ [8]; meta que puede considerarse baja si se compara con la del $95 \%$ del conglomerado de países de la UE y del $89 \%$ de Japón y de Estados Unidos [17], [23]. En aras de mejorar los índices de gestión sostenible de residuos de LLFU, algunas administraciones locales han tomado medidas como la combinación del modelo de REP con el modelo de impuestos, como el caso presentado por la administración distrital de Bogotá con la expedición del Decreto 442 de 2015, en el cual se establecen sanciones económicas a productores y usuarios de llantas que incumplan con lo establecido en la Resolución 1457 [24].

Tabla 1. Modelos de regulación para la gestión de residuos de LLFU [14, 15, 17-21].

\begin{tabular}{|l|l|l|}
\hline \multicolumn{1}{|c|}{ Modelo } & \multicolumn{1}{|c|}{ Característica } & $\begin{array}{l}\text { Países donde se practica y año de adopción } \\
\text { de la legislación }\end{array}$ \\
\hline & $\begin{array}{l}\text { Las tareas de recolección y } \\
\text { recuperación/reciclaje de los } \\
\text { Responsabilidad } \\
\text { Productor (REP). } \\
\text { los productores o importadores. }\end{array}$ & $\begin{array}{l}\text { Finlandia, Hungria, Italia, Lituania, Letonia, } \\
\text { Holanda, Noruega, Polonia, Rumania, España, } \\
\text { Suecia, Bélgica, Francia, Portugal (1999, 2000), } \\
\text { Estados Unidos (2000), Japón (2001), Taiwán } \\
\text { (1989), Corea del Sur (2003), Colombia (2010), } \\
\text { Brasil (2009), Ecuador (2013), Uruguay (2015). }\end{array}$ \\
\hline
\end{tabular}

Continúa $\rightarrow$ 


\begin{tabular}{|l|l|l|}
\hline \multicolumn{1}{|c|}{ Modelo } & \multicolumn{1}{c|}{ Característica } \\
$\begin{array}{l}\text { Cobro de multas } \\
\text { o impuestos a } \\
\text { los productores/ } \\
\text { importadores. }\end{array}$ & $\begin{array}{l}\text { Con los fondos recolectados } \\
\text { se financian organizaciones } \\
\text { encargadas de la recolección } \\
\text { y recuperación/reciclaje de los } \\
\text { residuos. }\end{array}$ & $\begin{array}{l}\text { Países donde se practión } \\
\text { de la legislación }\end{array}$ \\
\hline $\begin{array}{l}\text { Sistema de } \\
\text { mercado libre. }\end{array}$ & $\begin{array}{l}\text { Fomeno Unido (1999, 2000). } \\
\text { que obtengan beneficios } \\
\text { económicos a partir de la } \\
\text { utilización de los desechos. }\end{array}$ & $\begin{array}{l}\text { Dinamarca, Eslovaquia (1999, 2000), Japón } \\
\text { (2009), Estados Unidos (2007). }\end{array}$ \\
\hline
\end{tabular}

Fuente: elaboración propia.

Cabe resaltar que no ha transcurrido tiempo suficiente como para evaluar el impacto de las políticas implementadas en Colombia, pero puede inferirse que las administraciones locales deben esforzarse por buscar mecanismos que sean pertinentes para sus necesidades, seleccionando alternativas, pero también considerando la combinación de modelos exitosos en otras regiones. En otros países latinoamericanos, la gestión de residuos provenientes de las LLFU no cuenta con una legislación específica, sino que las LLFU se integran a la gestión de residuos sólidos especiales o peligrosos [25]. Desafortunadamente, en la literatura no se han reportado estudios orientados a identificar si este abordaje genera mejores resultados que tratar las LLFU con normatividades específicas, aspecto que permitiría contar con información confiable para seleccionar la regulación más conveniente.

El panorama descrito evidencia la necesidad de continuar buscando alternativas para el manejo de los desechos de caucho, con el fin de disminuir su impacto ambiental y, simultáneamente, buscar alternativas para que las empresas se acojan a la normatividad vigente en cada país. Además, la reutilización de residuos de caucho puede derivar en nuevas líneas de negocio para las empresas o incluso en nuevos emprendimientos, para lo cual la información presentada en este documento puede proporcionar algunos criterios para los estudios de viabilidad. La literatura publicada en los últimos años refleja un creciente interés por ahondar en el estudio de posibles aplicaciones para residuos de caucho, tanto para fabricar productos de bajo valor agregado, como para la obtención de artículos con altas especificaciones técnicas. Se percibe un esfuerzo por incursionar en nuevas aplicaciones y ampliar el abanico de posibilidades. Por lo anterior, en este trabajo se presenta una revisión sobre el uso de caucho reciclado en diversas áreas de la industria.

Con el fin de comprender las presentaciones disponibles del caucho reciclado y así profundizar luego en las aplicaciones de dicho material -lo cual constituye la temática central de este artículo-, se relacionan brevemente los principales métodos de reci- 
claje de caucho identificados durante la revisión bibliográfica realizada.

\section{PRINCIPALES MÉTODOS DE RECICLAJE DEL CAUCHO}

Los métodos de aprovechamiento de los residuos de caucho se clasifican en procesos mecánicos, criomecánicos, termo-mecánicos (molienda, mezclado de alta velocidad), químico-mecánicos (desvulcanización, proceso criogénico de Trelleborg [TCR, por sus siglas en inglés], hinchamiento en benceno), químicos (con reactivos orgánicos e inorgánicos), térmicos (en digestores, en autoclaves, en medios alcalinos, en medios neutros, con acción de vapor de alta presión), biotecnológicos, procesos con microondas y con ultrasonido [26], [27]. En la mayoría de estos procesos es necesario triturar el caucho hasta reducirlo a gránulos de tamaño y forma regular, y de esa manera facilitar que los aditivos químicos y agentes de expansión reaccionen adecuadamente con la estructura vulcanizada, a la vez que se separan más eficientemente las fibras de acero, textiles y aditamentos metálicos que puedan estar presentes en el caucho que se procesa.

En el caso de los procesos de trituración, los gránulos de caucho se comercializan en diferentes tamaños para que el cliente final seleccione el que sea más adecuado a sus necesidades. Si bien en la mayoría de las aplicaciones las propiedades del caucho reciclado triturado no logran igualar las del caucho virgen, el uso del primero presenta las siguientes ventajas respecto al caucho virgen, adicionalmente a la mencionada dis- minución del impacto ambiental cuando el caucho es reciclado [17], [28]:

- Su costo puede llegar a ser menos de la mitad de lo que cuesta el caucho virgen, si se compara con caucho natural o con caucho sintético.

- El módulo elástico o la resistencia al hinchamiento por solventes pueden ser superiores a las del caucho virgen [26].

- Las actividades de reciclaje generan empleo, principalmente mano de obra no calificada, especialmente en los países en vía de desarrollo [28].

Una alternativa para el uso de caucho reciclado por trituración mecánica consiste en incorporarlo a mezclas con caucho virgen, en las cuales este último actúa como matriz. El uso de caucho triturado en mezclas caucho virgen-caucho reciclado presenta algunas ventajas respecto al procesamiento de caucho virgen, entre las cuales se destacan las siguientes:

- Menor tiempo de proceso y menor consumo de energía durante el mezclado: durante las operaciones de trituración el caucho reciclado sufre una plastificación debido al trabajo mecánico al que es sometido; por lo tanto, el proceso de mezclado se facilita cuando se usa caucho reciclado respecto al mezclado de caucho virgen, y con menor calentamiento de los molinos.

- Mayor procesabilidad por calandrado y extrusión: cuando se fabrican artículos con estos métodos empleando mezclas de caucho recuperado y caucho virgen, se requieren temperaturas menores que cuando se procesa caucho virgen, lo cual 
facilita el proceso y aumenta la producción. Además, como efecto de la fase gel entrecruzada en el caucho reciclado, la mezcla presenta un comportamiento menos viscoelástico que el caucho virgen, lo cual facilita el control dimensional durante del procesamiento.

- Mejoramiento de la vulcanización y la resistencia al envejecimiento: el caucho reciclado impide la exudación del azufre que se puede presentar en materiales recientemente vulcanizados o crudos. Además, las mezclas que emplean caucho reciclado vulcanizan más rápidamente que las de caucho virgen; de esta manera se economiza tiempo y energía durante el proceso de vulcanización [27], [29].

Cuando el reciclaje se realiza por métodos químicos, biotecnológicos o por microondas, el resultado de los procesos son compuestos que pueden ser usados en las aplicaciones que se describen posteriormente en este documento.

Algunos estudios muestran que, bajo las condiciones adecuadas, la utilización de caucho obtenido a partir de LLFU recicladas o artículos genéricos fabricados con caucho (general rubber goods) [15], [30] no es perjudicial ni para la salud humana, ni para el medio ambiente [31], características funcionales que son atractivas para fomentar su uso.

\section{APLICACIONES DEL CAUCHO RECICLADO}

Por los desafíos técnicos y económicos inherentes a los procesos de reciclaje de los cauchos, hay que advertir que infortunada- mente la generación de desechos de residuos de caucho excede sus usos potenciales [16], pero a pesar de la complejidad de algunos de los procesos de reciclaje de caucho, es tan necesario encontrar soluciones que se siguen buscando aplicaciones para este tipo de desechos.

Dicha búsqueda para el caucho reciclado debe orientarse a obtener productos con mayor valor agregado, para que esta alternativa planteada sea económicamente atractiva, tenga mayores posibilidades de comercialización y, consecuentemente al aumentar los volúmenes de utilización de caucho reciclado, se disminuya el impacto ambiental de la disposición de los residuos de caucho, como se ha resaltado insistentemente en este documento. Las principales aplicaciones para el caucho reciclado reportadas en la literatura se presentan en la Tabla 2.

En el estado del arte que se muestra en este trabajo no se considera la incineración de residuos de caucho para producir vapor, electricidad o usarlos como combustible para hornos de cemento, puesto que se trata de alternativas técnicamente viables, pero que exigen un alto capital de inversión y por lo tanto solo están al alcance de grandes corporaciones u organismos multilaterales. Otra alternativa no considerada en esta investigación es la recuperación del negro de humo presente en residuos de caucho, lo cual disminuye los costos de los procesos de corte y trituración, pero es un proceso que genera negro de humo más costoso y de menor calidad que aquel que proviene de la refinación del petróleo. Además, se tiene el agravante de que la incineración de las LLFU exige equipos altamente especializados y 
costosos para evitar que se genere un efecto nocivo en el medio ambiente e implica bus- car alternativas para el uso de los otros componentes de las formulaciones de caucho.

Tabla 2. Resumen de las aplicaciones del caucho reciclado revisadas

\begin{tabular}{|c|c|}
\hline Tipo de aplicación & Referencias y año de publicación \\
\hline $\begin{array}{l}\text { Incineración para producir vapor, electricidad o como } \\
\text { combustible en hornos de cemento. }\end{array}$ & [16], [80]: 2001 \\
\hline $\begin{array}{l}\text { Recuperación del negro de humo presente en el caucho } \\
\text { reciclado. }\end{array}$ & [16] [80]:2001 \\
\hline \multicolumn{2}{|l|}{ Construcción e infraestructura } \\
\hline Asfaltos, pavimentos y concretos. & \begin{tabular}{|l} 
[10]: 2004, [40]: 2009, [33]: 2011, [11], [32], \\
[35]:2 014, [36], [37], [38], [39], [40]: 2015
\end{tabular} \\
\hline $\begin{array}{l}\text { Pisos para áreas recreativas y superficies. deportivas, pisos de } \\
\text { piscinas, pistas de boliche. }\end{array}$ & [36], [37], [38]: 2015 \\
\hline Aisladores sísmicos. & [42]: 2014 \\
\hline Aisladores acústicos. & [45]: 1998, [46]: 2007, [47]: 2011 \\
\hline \multicolumn{2}{|l|}{ Cuidado y recuperación del medio ambiente } \\
\hline Tratamientos de agua y soluciones acuosas. & $\begin{array}{l}\text { [60]: 2001, [56]: 2002, [57]: 2004, [58], [61]: } \\
\text { 2005, [53]: 2006, [63]: 2008, [59] [64] [70]: } \\
\text { 2009, [55] [62]: 2011, [54]: 2012, [69]: } 2013\end{array}$ \\
\hline Techos ecológicos (verdes). & $\begin{array}{l}\text { [50]: 2006, [48]: } 2010 \text { [49], [51]: 2012, [52]: } \\
2014\end{array}$ \\
\hline $\begin{array}{l}\text { Fabricación de celdas de combustible para generación de } \\
\text { energía. }\end{array}$ & [65]: 2011 \\
\hline \multicolumn{2}{|l|}{ Materiales compuestos de matriz polimérica para usos diversos } \\
\hline Implementos deportivos. & [36], [37], [38]: 2015 \\
\hline $\begin{array}{l}\text { Amortiguadores de impacto en muelles marítimos, } \\
\text { almohadillas para rieles y maquinaria industrial. }\end{array}$ & [69]: 2013, [36], [37]: 2015 \\
\hline $\begin{array}{l}\text { Industria automovilística: parachoques, guardabarros, tapetes } \\
\text { juntas y sellos de carrocería. }\end{array}$ & [36], [37] [38]: 2015 \\
\hline Equipos de minería. & [37]: 2015 \\
\hline Calzado y vestimenta. & [78]: 1993, [79]: 1996, [36]: 2015 \\
\hline Elaboración de adhesivos y sellantes. & [36]: 2015 \\
\hline Termoplásticos elastoméricos. & $\begin{array}{l}\text { [72]: 2009, [75]: 2011, [76]: 2012, [73], [74]: } \\
\text { 2013, [77]: } 2015\end{array}$ \\
\hline
\end{tabular}

Fuente: elaboración propia. 
A continuación se describen las aplicaciones del caucho reciclado que constituyen una alternativa, tanto para pequeñas y medianas empresas, como para grandes empresas.

\subsection{Construcción e infraestructura}

El caucho reciclado puede ser utilizado como componente de pavimentos y concretos para la construcción de vías y edificaciones. Emplear residuos de caucho en este tipo de aplicaciones representa, además de las importantes ventajas ambientales y económicas expuestas previamente, mejoras técnicas en este tipo de productos, como el incremento de la resistencia al impacto y la resistencia a la fatiga, lo que acarrea, sin embargo, algunas pérdidas en propiedades como el módulo elástico y la resistencia a la compresión [32]-[35]. Debido a lo anterior, el uso de caucho reciclado en concretos y pavimentos presenta retos económicos, ecológicos y técnicos que actualmente son tema de interés [33]. En el sector de la construcción también se utiliza el caucho reciclado para la fabricación de pisos antideslizantes, bases de tapetes, compuestos impermeables para techos y paredes. El asfalto modificado con caucho ha servido como base para pistas de hípica, drenajes de subsuelo y acondicionadores de suelo [36]-[38].

En una investigación orientada a la modificación de asfalto para pavimentos, se evaluó un sistema de polietileno lineal de baja densidad (LLDPE) con caucho reciclado triturado (CRT) [39], en el cual se realizó un proceso de desvulcanización dinámica del CRT durante el mezclado de ambos componentes en extrusora de doble husillo. Posteriormente, se llevó a cabo un nuevo proceso de vulcanización dinámica sobre la mezcla, para luego ser incorporada en asfalto. Según los autores, el proceso de desvulcanización durante el mezclado en extrusora permite mantener casi completamente la elasticidad original del caucho y mejora su dispersión en la matriz termoplástica; a su vez, el proceso posterior de vulcanización mejora las interacciones entre las partículas de caucho y la matriz de LLDPE, lo que aumenta el desempeño de propiedades del asfalto modificado, como la sensibilidad térmica y la resistencia a la penetración.

En otro estudio se investigó experimentalmente la posibilidad de incorporar caucho reciclado proveniente de LLFU a mezclas de concreto compactado con rodillos para pavimentos. Los resultados mostraron que mediante este proceso es posible obtener un compuesto con mejores propiedades de consistencia, ductilidad y resistencia al agrietamiento y menor densidad específica y absorción de agua, pero algunas propiedades mecánicas disminuyeron. Acá proponemos que este nuevo material podría ser utilizado en vías de bajo tráfico, caminos rurales y amplias áreas para pedestres [11]. Otros estudios han evidenciado que la incorporación de caucho reciclado triturado también es factible en matrices de cemento Portland, así como mezclas ligantes usadas para construcciones con ladrillos y concreto. Sin embargo, en la mayoría de las investigaciones se ha planteado que a pesar de la reducción de densidad y la mejoría en propiedades de resistencia al impacto, tenacidad, ductilidad y aislamiento acústico, también se puede generar una disminución en propiedades como resistencia a la com- 
presión y a la tracción [10], lo que evidencia la necesidad de continuar buscando soluciones para superar este tipo de inconvenientes. Yilmaz y Degirmenci [40] desarrollaron un compuesto de cemento Portland al que se incorporó caucho reciclado molido y cenizas provenientes de la quema de carbón, y lo evaluaron experimentalmente mediante ensayos de flexión, compresión y absorción de agua. Los resultados mostraron que se logra atenuar la reducción en las propiedades de compresión generadas por la incorporación de partículas de caucho reciclado molido, si simultáneamente se incorporan cenizas a la formulación; esto le confiere a la mezcla un mayor rango de aplicaciones potenciales en el sector de la construcción.

Otro compuesto desarrollado para este tipo de aplicaciones es el concreto con incorporación de agregados de caucho reciclado reforzado con fibras de acero. Este compuesto mostró que cuando se utiliza una proporción óptima de caucho reciclado y simultáneamente fibras de acero, se puede obtener un concreto viable para el uso en aplicaciones de ingeniería civil, ya que continúa presentando las buenas propiedades de ductilidad y tenacidad de la mezcla original, con consecuencias menos drásticas sobre la resistencia a la compresión y a la flexión [41].

En las grandes ciudades, son cada vez más importantes los sistemas de aislamiento sísmico, que generalmente están constituidos de materiales de gran tamaño, peso y alto costo, dado que se trata de estructuras compuestas por placas de acero exteriores unidas por placas reforzantes interiores elastoméricas. En el caso de gran- des construcciones, es más fácil financiar los altos costos de este tipo de sistemas aislantes; sin embargo, los costos son inviables en el caso de construcciones livianas, por lo que conviene buscar materiales que desempeñen esa función con un menor impacto económico. Un estudio demostró que soportes de amortiguación de vibraciones reforzados con fibras y con placas interiores de caucho reciclado como elastómero podrían desempeñarse como sistemas de aislamiento sísmico eficientes en construcciones livianas (residenciales o comerciales de baja altura). Mediante cálculos teóricos y pruebas experimentales, se demuestra que dichos materiales pueden mantener la estabilidad de la estructura aceptando un mayor desplazamiento lateral, y aumentar la razón de disipación de energía, hecho que potencialmente viabiliza su utilización en este tipo de edificaciones [42].

De la revisión de la literatura se infiere que en el área de construcción e infraestructura existen alternativas viables y económicamente atractivas para los residuos de caucho, lo cual constituye una posibilidad de aplicación con valor agregado para este tipo de desechos en países como Colombia, que viven un crecimiento significativo de la industria de la construcción [43] o en la recuperación de vías terciarias, que comprenden alrededor del $70 \%$ de la red vial del país [44]. Para impulsar decididamente el uso de caucho reciclado en ese tipo de aplicaciones, es de vital importancia la adecuada capacitación del recurso humano directamente involucrado, el acatamiento de la legislación ambiental y de buenas prácticas de manufactura [22], así como la evaluación de requisitos técnicos pertinentes que otorguen seguridad para la aplicación de 
estos residuos, desafíos que deben ser abordados a corto plazo.

En la actualidad, el ruido potencialmente contaminante representa un serio y complejo problema, debido a la necesidad de contar con ambientes sanos y con la menor contaminación acústica posible; de esta manera, actualmente se buscan materiales delgados, livianos y de bajo costo que absorban ondas acústicas en amplias frecuencias. En algunos estudios se ha evaluado la capacidad de absorción acústica de partículas de caucho reciclado, y se ha mostrado la potencial aplicación de dicho material en esta área [45]. Algunos autores han concluido que se pueden usar materiales compuestos que contienen partículas de caucho reciclado para aplicaciones donde se busque absorción acústica, gracias a la capacidad comprobada del caucho para absorber sonido de baja frecuencia, de tal manera que si se complementa con un material que absorba ondas acústicas de alta frecuencia se tendría una importante barrera contra el ruido utilizando materia prima de alta disponibilidad y bajo costo [46], además de desempeñarse igual o incluso mejor que muchos de los materiales que se comercializan actualmente para este tipo de productos [47].

\subsection{Cuidado y recuperación del medio ambiente}

En lo concerniente al cuidado y recuperación del medio ambiente, se ha demostrado la utilidad del caucho reciclado como componente para la fabricación de techos verdes, como adsorbente de sustancias tóxicas presentes en soluciones acuosas, en sistemas de filtración y limpieza de aguas residuales y derrames, y como componente de celdas de combustible. A continuación se describen estas aplicaciones con mayor detalle.

Los techos ecológicos, también conocidos como techos verdes, son llamados de esta manera porque cuando se cubren con vegetación se facilita un medio de crecimiento para las plantas y se tornan de color verde [48]. Al evaluar la configuración de esas estructuras desde la capa exterior hacia la interior, se encuentra que están compuestas de vegetación, sustrato, filtro, drenaje, protección, barrera de raíces y una capa impermeable; sin embargo, los materiales usados normalmente no son amigables con el medio ambiente, pues se trata de termoplásticos como polipropileno, polietileno, poliésteres y policloruro de vinilo (PVC) [49]. En la mayoría de las configuraciones, la capa de drenaje está compuesta de agregados de materiales de arenisca, principalmente puzolana, cuya extracción, procesamiento y disposición final genera un gran impacto medioambiental. Esto hace evidente la pertinencia de sustituirlas con materiales con menor impacto ambiental, como el caucho reciclado triturado, y lleva además a una considerable disminución del peso del techo [50], [51]. Gracias al análisis del ciclo de vida completo de los techos verdes con caucho reciclado se encontró que el uso de este tipo de materiales tiene un impacto ambiental hasta del $7 \%$ menor en relación con aquellos techos que utilizan materiales convencionales, como la puzolana [52].

El caucho de las LLFU ha probado ser un medio interesante y económicamente atractivo para la absorción de contaminantes tó- 
xicos provenientes de soluciones acuosas. Además, la conversión de LLFU en carbón activado para uso potencial en tratamientos de aguas desechadas con presencia de contaminantes tóxicos posee un componente social bastante atrayente. Esto no solo provee un método para el manejo de los residuos sólidos generados, sino que también reduce en gran medida el costo de producción de este tipo de adsorbentes. Entre el 25 y el $30 \%$ del peso de una llanta está constituido por negro de humo, que a su vez posee un contenido de entre 70 y $75 \%$ de carbono [53]. Este adsorbente carbonáceo obtenido de la pirolisis de LLFU es bastante similar al carbón activado, y mediante tratamiento con calor y sometimiento a atmósferas de dióxido de carbono o vapor se puede lograr la activación de su superficie; con esto se aumenta su área superficial y porosidad, y consecuentemente se mejora su comportamiento como adsorbente [54].

Algunos estudios han demostrado la aplicación exitosa de los desechos de caucho proveniente de LLFU como adsorbentes para varios tipos de contaminantes, entre los cuales se encuentran derivados del petróleo como xileno y tolueno [55], colorantes [56], pesticidas [57], fenoles [58], iones metálicos como el cobre de valencia II [59], el cromo de valencia IV [60], mercurio [61], cadmio [62], plomo [63] y níquel [54].

Un nuevo sistema de filtración para tratar aguas residuales de lastre desarrollado con caucho triturado filtra efectivamente los contaminantes presentes en este tipo de aguas, con menor obstrucción en relación con los filtros de arena y, además, con mejor capacidad de adsorción orgánica y mayor área superficial [64]; esto hace que se torne en un material promisorio para este proceso de filtración.

Las celdas de combustible microbianas son una aplicación que gana cada vez más terreno dentro de las tecnologías de generación de energía; sin embargo, actualmente poseen retos como la reducción del costo de sus materiales para poder viabilizar su producción a gran escala y ampliar su aplicabilidad. Los materiales convencionales incluyen gránulos de grafito, papel, mallas y telas de carbón, y carbón activado, los cuales poseen costos entre $50 \mathrm{US} \$ / \mathrm{m}^{2} \mathrm{y}$ $1000 \mathrm{US} \$ / \mathrm{m}^{2}$, lo que hace inviable su uso a gran escala [65]. En un estudio realizado por Wang et al. [65] se evaluó la factibilidad del uso de caucho reciclado granulado con un recubrimiento conductor como ánodo de celdas de combustible microbianas. Los autores encontraron que las partículas de caucho reciclado provenientes de LLFU presentan buena conductividad cuando son recubiertas con dos a cuatro capas de grafito y su área superficial es mayor a la de los gránulos de grafito, lo que favorece la fijación de microbios en su superficie. Los costos de este tipo de ánodo son mucho menores a los de los materiales convencionales, ya que cuestan entre $0,71 \mathrm{US} \$ / \mathrm{m}^{2}$ y $1,42 \mathrm{US} \$ / \mathrm{m}^{2}$ con el proceso de recubrimiento incluido, aspecto que evidencia la potencial aplicación del caucho en este tipo de tecnologías.

Se han patentado compuestos de matriz polimérica de poliuretano y fase dispersa de caucho reciclado [66]-[68]. Con esto se encuentra que una interesante aplicación de este tipo de compuestos, pero que hasta el momento solo ha sido probada a escala de 
laboratorio, es la fabricación de bandejas flotantes con plantas para que las raíces efectúen tratamiento de aguas de lagos, estanques y cuencas contaminados por desechos de la industria minera [69].

Existen algunas resinas absorbentes que se usan para limpiar el agua cuando hay derrames oleosos durante el transporte de diversos componentes de la industria petrolera, sin embargo, su uso es bastante limitado, debido principalmente a sus altos costos. Wu y Zhou [70] utilizaron partículas de caucho triturado reciclado (CTR) para realizar un proceso de mezclado con un monómero de 4-tert-butilestireno a través de una reacción de copolimerización por injerto, fabricando absorbentes de compuestos oleosos. Los materiales desarrollados absorben eficazmente el crudo de acuerdo con la norma ASTM F726 [71], y aunque mostraron un desempeño un poco inferior a los productos comerciales utilizados para este fin, sus costos son significativamente más bajos, lo cual compensaría la falencia mencionada anteriormente.

\subsection{Materiales compuestos de matriz polimérica para usos diversos}

Una forma de reciclaje de caucho proveniente de LLFU que parece bastante promisoria es su incorporación con forma de material particulado en matrices poliméricas para obtener materiales compuestos, los cuales son bastante apetecidos en la industria actual, debido a que combinan propiedades de ambos componentes para obtener materiales con propiedades intermedias o sinergia de estas en el producto final. Cada tipo de industria tiene una motivación diferente para emplearlos, de manera que en los termoestables se busca mejorar la tenacidad; en los termoplásticos el objetivo es obtener elastómeros termoplásticos, mientras que la industria del caucho trata de encontrar cargas de relleno de menor costo [17].

En la actualidad se fabrican de manera exitosa pedales y volantes de automóviles empleando compuestos de polipropileno/ caucho reciclado proveniente de desechos de LLFU. Algunos investigadores han realizado estudios que han tratado los residuos de caucho con bitumen para mejorar las propiedades de alargamiento a la rotura, estabilidad térmica y procesabilidad de los compuestos de matriz de PP con incorporación de dicho residuo [72].

Al-Malki et al. [73] realizaron mezclas de polietileno de alta densidad (HDPE) con caucho de LLFU reciclado triturado (CLRT) y de HDPE con caucho polibutadieno (PB) virgen, y compararon las propiedades mecánicas y reológicas de las mezclas. El comportamiento mecánico del CLTR fue muy similar al del PB virgen en la matriz de HDPE, lo cual representa una oportunidad para el uso de caucho reciclado en ese tipo de matrices.

A pesar de ser un campo promisorio para la utilización de residuos de caucho, la incorporación de CRT en matrices poliméricas vírgenes plantea un reto que aún es objeto de estudio: la compatibilidad entre los componentes. Una solución estudiada es el uso de adhesivos, principalmente resinas termoestables y matrices termoplásticas, para mejorar la compatibilidad de las partículas de CRT con la matriz, pero el desempeño que se ha 
obtenido no es aún el más apropiado. Ante esta dificultad, una alternativa es la modificación de la superficie de las partículas de CRT para crear puntos específicos de interacción entre las fases y así mejorar la transferencia de cargas desde la matriz hacia la fase de caucho, lo cual a su vez ayuda a optimizar algunas propiedades mecánicas. Estas modificaciones pueden obtenerse a través de medios físico-mecánicos o químicos, siendo la activación superficial, la desvulcanización, la vulcanización dinámica y la compatibilización algunos de los procesos más utilizados para este fin [39], [74] y [75].

Mészáros, Bárány y Czvikovszky [76] utilizaron tratamiento de irradiación de alta energía para activar la superficie de partículas de CRT con la intención de mejorar su compatibilidad con una matriz de polietileno lineal de baja densidad (LDPE) y etilen-vinil-acetato (EVA). La evaluación de las propiedades mecánicas mostró que para una composición fija de LDPE/EVA/CTR, el aumento en la cantidad de energía irradiada sobre los compuestos incrementa la resistencia a la tracción, la deformación de ruptura, la dureza Shore $\mathrm{D}$ y la tenacidad del compuesto. Los mejores desempeños observados en estas propiedades son atribuidos a la acción de la energía irradiada sobre las partículas de CTR, que activa algunas zonas localizadas de la superficie de las partículas de caucho, en las cuales se forman enlaces químicos entre el caucho, el EVA y el LDPE [76].

Lima et al. [77] demostraron que la incorporación de CTR en matrices termoplásticas es una alternativa viable para darle una aplicación de valor agregado a los residuos de este tipo. Los autores evaluaron experimen- tal y teóricamente las propiedades reológicas de mezclas ternarias de caucho etileno propileno (EPR)/CTR en matriz de polipropileno (PP) y encontraron que la inclusión de una fase elastomérica como el EPR, de baja viscosidad y alta afinidad con los demás componentes, mantiene un comportamiento pseudoplástico en el compuesto y permite contrarrestar el aumento en la viscosidad de la mezcla causado por la presencia de las partículas de CTR; esto le confiere procesabilidad adecuada para el moldeo por inyección que permite obtener piezas con mayor valor agregado.

Otra potencial aplicación para los compuestos de poliuretano y caucho reciclado mencionados en secciones anteriores es la fabricación de boyas flotantes capaces de soportar grandes compresiones entre embarcaciones y muelles mientras los barcos están atracados [69]. En este estudio se sintetizó el compuesto a nivel de laboratorio, y las pruebas realizadas con los prototipos del producto final determinaron que el desempeño fue satisfactorio.

En el sector calzado también se ha visto una probable aplicación del compuesto poliuretano/caucho reciclado. En la patente de Panaroni y McLellan [78], se propone la mezcla de partículas secas de caucho reciclado provenientes principalmente de LLFU pos consumo con poliol e isocianato para formar un precursor de poliuretano con alta resistencia al desgaste, adecuado para la fabricación de suelas y capelladas de botas.

Duan y Stammler [79] patentaron la invención de una dispersión acuosa compuesta de un pre polímero de poliuretano aniónico empleando partículas de caucho reciclado 
como agente entrecruzante. Dicho compuesto presenta propiedades adhesivas, buena resistencia al agua, estabilidad a bajos $\mathrm{pH}$ y buena resistencia térmica, lo que según los inventores hace viable la utilización de dichos adhesivos en el sector calzado.

\section{CONCLUSIONES}

En este trabajo se efectuó una revisión sobre las principales aplicaciones de caucho reciclado, siendo las LLFU la principal fuente de este residuo. Si bien el reciclaje y la utilización de estos materiales implican retos tecnológicos significativos, y en algunas condiciones los costos de implementación son altos, la necesidad de disminuir su impacto ambiental ha impulsado la realización de estudios sobre nuevas aplicaciones para ese tipo de desperdicios que viabilicen su uso desde el punto de vista económico y técnico.

Los países de la UE, Japón y Estados Unidos tienen el mayor desarrollo tecnológico y en legislación sobre la temática, pero independientemente del grado de desarrollo que tenga una región en esos dos ámbitos, hay que buscar alternativas para el caucho reciclado que permitan a los productores y comercializadores cumplir con las regulaciones sin afectar su rentabilidad, o generar nuevas líneas de negocio e incluso nuevos emprendimientos.

El reciclaje energético o la recuperación del negro de humo presente en las LLFU requieren grandes inversiones en infraestructura y sistemas de control de emisiones, por lo cual este estudio se centró en aplicaciones viables para medianas y pequeñas em- presas, bien sea de países desarrollados o de aquellos en vía de desarrollo.

Uno de los principales campos potenciales de aplicación para los residuos de caucho es utilizarlos como modificadores de pavimentos y concretos, y mejorar así algunas propiedades como liviandad y ductilidad, pero debe tener en consideración que algunas características funcionales como la resistencia a la compresión y a la flexión disminuyen cuando se agrega ese tipo de desechos. Además, se identificaron líneas de investigación con posibilidades de generar alto valor agregado, como el uso de caucho reciclado en sistemas de adsorción de contaminantes en medios acuosos, techos ecológicos y fabricación de celdas de combustible para generación de energía, que aún están en fase de investigación. El uso de caucho reciclado en mezclas con caucho virgen presenta algunas ventajas tecnológicas, ambientales y sociales con respecto al uso de caucho virgen, como menor costo, mayor facilidad en algunos procesos como calandrado y extrusión, mayor eficiencia en el proceso de vulcanización y reducción de consumo de energía.

El desarrollo de elastómeros termoplásticos que utilicen caucho reciclado parece ser un campo bastante promisorio por la pertinencia de obtener materiales con las principales propiedades de los elastómeros, pero con la facilidad de procesamiento de los termoplásticos, aunque aún se encuentra en fase de investigación y actualmente existen pocos productos comerciales de esta clase. La utilización de caucho posconsumo como componente para la fabricación de materiales compuestos con diversos tipos de poliu- 
retano posee un gran potencial para la producción de componentes de la industria del calzado (por ejemplo suelas, capelladas y adhesivos), lo que podría favorecer a una industria como el sector del calzado, que debe buscar alternativas para disminuir costos de producción. Adicional a esto, se considera que el caucho reciclado puede ser utilizado para una gran diversidad de aplicaciones ornamentales.

Los productos fabricados con caucho reciclado presentan en términos generales un desempeño inferior al de los que utilizan cauchos vírgenes, y para reducir esta diferencia es necesario mejorar la compatibilidad del caucho reciclado con las matrices a las cuales se adiciona. Para mejorar la compatibilidad se ha avanzado en la modificación de las partículas de caucho a través de métodos químicos y físico-mecánicos.

Se detectó que la mayoría de los estudios reportados se enfocan en la evaluación de propiedades mecánicas y muy pocos abarcan los temas de la procesabilidad, un factor fundamental para el desarrollo de productos con alto valor agregado. Se detectaron además falencias en investigaciones referentes a las propiedades térmicas, propiedades de largo plazo, de hinchamiento y dinámico-mecánicas de los cauchos reciclados y compuestos fabricados con ellos.

Es de resaltar que el estudio hecho permitió evidenciar cómo se viene investigando en una temática con unos retos científicos de alta relevancia, pero que ameritan la mayor atención por el problema ambiental significativo asociado al manejo de los residuos de caucho.

\section{AGRADECIMIENTOS}

Los autores agradecen al Sistema de Investigación, Desarrollo Tecnológico e Innovación (SENNOVA) por la financiación del proyecto "Desarrollo de materiales compuestos con residuos de caucho reciclado para el sector calzado, construcción liviana y decoración", que permitió la elaboración de este trabajo.

\section{REFERENCIAS}

[1] Y. Fang, M. Zhan y Y. Wang, "The Status of Recycling of Waste Rubber," Materials \& Design, vol. 22, no. 2, pp. 123-127, 2001. https://doi. org/10.1016/s0261-3069(00)00052-2

[2] Malaysian Rubber Board [MRB], "Archive: Natural Rubber Statistics 2015," 2015. [En línea]. Disponible en: http:// www.lgm.gov.my/nrstat/nrstats.pdf

[3] International Rubber Study Group [IRSG], "Latest World Rubber Industry Outlook now available from IRSG", 2014. [En línea]. Disponible en: http:// www.rubberstudy.com/news-article. aspx?id=5081\&b=earlier-news . aspx

[4] L. C. Alves, M. M. Rubinger, E. Tavares, J. Janczak et al., "Synthesis, Spectroscopic Characterization, Crystal Structure and Natural Rubber Vulcanization Activity of New Disulfides Derived form Sulfonyldithiocarbimates," Journal of Molecular Structure, vol. 1048, pp. 244251, 2013. https://doi.org/10.1016/j. molstruc.2013.05.062

[5] G. Peláez, S. Velásquez y D. Giraldo, "Aditivos para el Procesamiento del 
Caucho Natural y su Aplicación en Pequeñas Plantaciones de Caucho," Informador Técnico, vol. 78, no. 2, pp. 166-174, 2014.

[6] H. Manuel y W. Dierkes. Recycling Rubber. Shawbury. Shropshire, Inglaterra. R. Dolbey: Rapra Technology Limited, 1997.

[7] M. Sienkiewicz, J. Kucinska-Lipka, $\mathrm{JH}$. Janik y A. Balas, "Progress in used tyres management in the European Union: A review," Waste Management, vol. 32, no. 10, pp. 1742-1751, 2012. https://doi.org/10.1016/j.wasman.2012.05.010

[8] Ministerio de Ambiente, Vivienda y Desarrollo Territorial, "Resolución 1457": Por la cual se Establecen los Sistemas de Recolección Selectiva y Gestión Ambiental de Llantas Usadas y se Adoptan Otras Disposiciones. Bogotá, Colombia, Jul. 2010.

[9] Agence France Presse [AFP], "Iglús en Colombia? Sí, pero de llantas de desecho," 2015. [En línea]. Disponible en: http://www.elespectador.com/noticias/ nacional/iglus-colombia-si-de-llantas-de-desecho-articulo-551771

[10] R. Siddique y T. Naik, "Properties of Concrete Containing Scrap-tire Rubber - An Overview," Waste Managment, vol. 24, no. 6, pp. 563-569, 2004. https://doi. org/10.1016/j.wasman.2004.01.006

[11] A. Meddah, M. Beddar y A. Bali, "Use of Shredded Rubber Tire Aggregates for Roller Compacted Concrete Pavement," Journal of Cleaner Production, vol. 72, pp. 187-192, 2014. https://doi. org/10.1016/j.jclepro.2014.02.052
[12] European Tyre \& Rubber Manufacturers' Association [ETRMA], "Statics Booklet N. 6", 2014. [En línea]. Disponible en: http://www.etrma.org/uploads/ documents / $20150408 \% 20-\% 20$ Statistics\%20booklet\%202014\%20 FINAL\%20(modified).pdf

[13] M. Forrest. Recycling and Re-use of Waste Rubber. Shrewsbury, Reino Unido: Smithers Rapra Technology, 2014.

[14] M. Sienkiewicz, J. Kucinska-Lipka, H. Janik y A. Balas, "Progress in used tyres management in the European Union: $A$ review," Waste Management, vol. 32, no. 10, pp. 1742-1751, 2012. https://doi.org/10.1016/j.wasman.2012.05.010

[15] European Tyre \& Rubber Manufacturers' Association [ETRMA], "End of Life Tyres: A Valiable Resource with Growing Potential", 2010. [En línea]. Disponible en: http://www.etrma.org/ uploads/Modules/Documentsmanager/brochure-elt-2011-final.pdf

[16] Irevna, "Tire Recycling Industry: A Global View," s. f. [En línea]. Disponible en: https://www.yumpu.com/en/document/view/38732587/tire-recycling-industry-global-research-amp-analytics

[17] S. Ramarad, M. Khalid, C. Ratnam, A. Chuah y W. Rashmi, "Waste tire rubber in polymer blends: A review on the evolution, properties and future," Progress in Material Science, vol. 72, pp. 100140, 2015. https://doi.org/10.1016/j. pmatsci.2015.02.004

[18] J. Chong, L. Mason, S. Pillora y D. Giurco. Product Stewardship Schemes in Asia: China, South Korea, Japan and 
Taiwan. Sydney, Australia: Institue for Sustainable Futures, University of Technology Sydney, 2009.

[19] Conselho Nacional do Meio Ambiente [Conama], "Resolução no. 416 de 2009”, 2009. Brasilia, Brasil. [En línea]. Disponible en: http://www.mma.gov. $\mathrm{br} /$ port/conama/legiabre.cfm?codle$g i=616$

[20] Ministerio del Ambiente, "Acuerdo no. 020”, 2013. Quito, Ecuador. [En línea]. Disponible en: http://extwprlegs1.fao. org/docs/pdf/ecu139932.pdf

[21] Ministerio de Vivienda, Ordenamiento Territorial y Medio Ambiente [MVOTMA], "Decreto 358 de 2015," 2015. Montevideo, Uruguay. [En línea]. Disponible en: https://www.impo.com.uy/ bases/decretos/358-2015

[22] V. Ramírez y J. Antero, "Evolución de la Normativa Ambiental Colombiana en Función de las Tendencias Mundiales de Desarrollo Sostenible," Revista NOOS, vol. 3, no. 4, pp. 34-55, 2013.

[23] C. Lagarinhos y J. Tenório, "Logística reversa dos pneus usados no Brasil," Polímeros, vol. 23, no. 1, pp. 49-58, 2013.

[24] Colombia, Alcaldía de Bogotá, "Decreto 442 de 2015": Por medio del cual se crea el Programa de aprovechamiento y/o valorización de llantas usadas en el Distrito Capital y se adoptan otras disposiciones, 2015. Bogotá, Colombia. [En línea]. Disponible en: http:// www.alcaldiabogota.gov.co/sisjur/ normas/Norma1.jsp?i=63644
[25] Federación Interamericana del Cemento [FICEM], "Marco Normativo sobre Coprocesamiento y Gestión de Residous en Iberoamérica," 2009. [En línea]. Disponible en: http://www. ficem.org/ficem/temas-clave/recuperacion-de-residuos/marco-normativo-sobre-coprocesamiento.html

[26] E. Abraham, B. Cherian, P. Elbi, L. Pothen y S. Thomas, "Recent Advances in the Recycling of Rubber Waste," en A. Fainleib y 0 . Grigoryeva, Recent Developments in Polymer Recycling. Kerala, India. Transworld Research Network. pp. 47-100, 2011.

[27] B. Adhikari, D. De y S. Maiti, "Reclamation and Recycling of Waste Rubber," Progress in Polymer Science, vol. 25, no. 7, pp. 909-948, 2000. https://doi. org/10.1016/s0079-6700(00)00020-4

[28] The Schumacher Centre for Technology \& Development, "Practical Action: Recycling Rubber," s. f. [En línea]. Disponible en: http://practicalaction.org/ media/preview/10541

[29] V. Rajan, W. Dierkes, R. Joseph y J. Noordermeer, "Science and Technology of Rubber Reclamation with Special Attention to NR-based Waste Latex Products," Progress in Polymer Science, vol. 31, no. 9, pp. 811-834, 2006. https://doi.org/10.1016/j.progpolymsci.2006.08.003

[30] L. Rincón, J. Coma, G. Pérez, A. Castell, D. Boer y L. Cabeza, "Environmental Performance of Recycled Rubber as Drainage Layer in Extensive Green Roofs. A Comparative Life Cycle Assessment," 
Building and Environment, vol. 74, pp. 22-30, 2014. https://doi.org/10.1016/j. buildenv.2014.01.001

[31] Instituto de Biomécanica de Valencia [IBV], "Study of the Incidence of Recycled Rubber from Tyres in Environment and Human Health", s. f. [En línea]. Disponible en: http://www.isss-sportsurfacescience.org/downloads/documents/muw7lmt0wh_ibv.pdf

[32] Y. C. Guo, J. H. Zhang, G. Chen, G. M. Chen y Z. H. Xie, "Fracture Behaviors of a New Steel Fiber Reinforced Recycled Aggregate Concrete with Crumb Rubber," Construction and Building Materials, vol. 53, pp. 32-39, 2014. https://doi.org/10.1016/j.conbuildmat.2013.11.075

[33] K. San Song, I. Hajirasouliha y K. Pilakoutas, "Strength and Deformability of Waste Tyre Rubber-filled Reinforced Concrete Columns," Construction and Building Materials, vol. 25, no. 1, pp. 218226, 2011. https://doi.org/10.1016/j. conbuildmat.2010.06.035

[34] A. Khaloo, M. Dehestani y P. Rahmatadabi, "Mechanical properties of concrete containing a high volume of tire-rubber particles," Waste Management, vol. 28, no. 12 , pp. 2472-2482, 2008. https://doi. org/10.1016/j.wasman.2008.01.015

[35] X. Shu y B. Huang, "Recycling of Waste Tire Rubber in Asphalt and Portland Cement Concrete: An Overview," Construction and Building Materials, vol. 67, pp. 217-224, 2014. https://doi.org/10.1016/j.conbuildmat.2013.11.027
[36] Universe Projects, "Applications for Recycled Rubber", s. f. [En línea]. Disponible en: http://www.universe-projects.com/technologies/tire-recycling/ applications-for-recycled-rubber/. [URL desabilitado].

[37] Valorpneu, "Informação Técnica: Soluções para Pneus Usados: Reutilização para Outros Fins," s. f. [Em línea]. Disponible en: http://valorpneu.pt/artigo.aspx?lang=pt\&id_object $=224 \&$ name $=$ Reutilizacao-para-Outros-Fins

[38] Micor Recycling Ltd., "Knowledge Center," s. f. [En línea]. Disponible en: http:// www.micorrecycling.com/knowledge. php

[39] S. Wang, Q. Wang, X. Wu y Y. Zhang, "Asphalt modified by thermoplastic elastomer based on recycled rubber," Construction and Building Materials, vol. 93, pp. 678-684, 2015. https://doi.org/10.1016/j.conbuildmat.2015.06.047

[40] A. Yilmaz y N. Degirmenci, "Possibility of Using Waste Tire Rubber and Fly Ash with Portland Cement as Construction Materials," Waste Managment, vol. 29, no. 5, pp. 1541-1546, 2009. https://doi. org/10.1016/j.wasman.2008.11.002

[41] X. Jian-he, G. Yong-Chang, L. Li-sha y $X$. Zhi-hong, "Compressive and Flexural Behaviours of a New Steel-fibre-reinforced Recycled Aggregate Concrete with Crumb Rubber," Construction and Building Materials, vol. 79, pp. 263-272, 2015. https://doi.org/10.1016/j.conbuildmat.2015.01.036 
[42] M. Spizzuoco, A. Calabrese y G. Serino, "Innovative Low-cost Recycled Rubberfiber Reinforced Isolator: Experimental Tests and Finite Element Analyses," Engineering Structures, vol. 76, pp. 99111, 2014. https://doi.org/10.1016/j. engstruct.2014.07.001

[43] S. Clavijo, A. Vera y N. Vera, "La inversión en infraestructura en Colombia 20122020," Carta Financiera ANIF, vol. 00, no. 0161, pp. 11-19, 2013.

[44] Ministerio de Transporte, Departamento Nacional de Planeación [DNP], "Plan de vías compatibles con el clima," Bogotá, 2014. [En línea]. Disponible en: http://www.minambiente. gov.co/images/cambioclimatico/ pdf/Plan_nacional_de_adaptacion/ Plan_V\%C3\%ADas-CC_V\%C3\%ADas_ Compatibles_con_el_Clima.pdf

[45] J. Pfretzschner y R. Rodríguez, "Acoustic Properties of Rubber Crumbs," Polymer Testing, vol. 18, no. 2, pp. 81-92, 1998. https://doi.org/10.1016/S01429418(98)00009-9

[46] Z. Hong, L. Bo, H. Guangsu y H. Jia, "A Novel Composite Sound Absorber with Recycled Rubber Particles," Journal of Sound and Vibration, vol. 304 no. 1-2, pp. 400-406, 2007. https://doi. org/10.1016/j.jsv.2007.02.024

[47] R. Maderuelo-Sanz, M. Martín-Castizo y R. Vílchez-Gómez, "The Performance of Resilient Layers Made from Recycled Rubber Fluff for Impact Noise Reduction," Applied Acoustics, vol. 72, no. 11, pp. 823-828, 2011. https://doi. org/10.1016/j.apacoust.2011.05.004
[48] J. Czemiel Berndtsson, "Green roof performance towards management of runoff water quantity and quality: $A$ review," Ecological Engineering, vol, 36, no. 4, pp. 351-366, 2010. https://doi.org/10.1016/j.ecoleng.2009.12.014

[49] G. Pérez, A. Vila, L. Rincón, C. Solé y L. Cabeza, "Use of Rubber Crumbs as Drainage Layer in Green Roofs as Potential Energy Improvement Material," Applied Energy, vol. 97, pp. 347-354, 2012. https://doi.org/10.1016/j.apenergy.2011.11.051

[50] M. Anderson, K. Kirkland, T. Guidotti y C. Rose, "A Case Study of Tire Crumb Use on Playgrounds: Risk Analysis and Communication when Major Clinical Knowledge Gaps Exist," Environmental Health Perspectives, vol. 114, no. 1, pp. 1-3, 2006. https://doi. org/10.1289/ehp.7629

[51] L. Solano, A. Ristvey, J. Lea-Cox y S. Cohan, "Sequestering Zinc from Recycled Crumb Rubber in Extensive Green Roof Media," Ecological Engineering, vol. 47, pp. 284-290, 2012. https://doi. org/10.1016/j.ecoleng.2012.07.002

[52] L. Rincón, J. Coma, G. Pérez, A. Castell, D. Boer y L. Cabeza, "Environmental Performance of Recycled Rubber as Drainage Layer in Extensive Green Roofs. A Comparative Life Cycle Assessment," Building and Environment, vol. 74, pp. 22-30, 2014. https://doi. org/10.1016/j.buildenv.2014.01.001

[53] R. Murillo, E. Aylón, M.V. Navarro, M.S. Callén, A. Aranda, A.M. Mastral, " The application of thermal processes to 
valorise waste tyre," Fuel Processing Technology, vol. 87, no. 2, pp. 143147, 2006. https://doi.org/10.1016/j. fuproc.2005.07.005

[54] V. Gupta, M. Ganjali, A. Nayak, B. Bhushan y S. Agarwal, "Enhanced Heavy Metals Removal and Recovery by Mesoporous Adsorbent Prepared from Waste Rubber Tire," Chemical Engineering Journal, vol. 197, pp. 330-342, 2012. https:// doi.org/10.1016/j.cej.2012.04.104

[55] L. Alamo-Nole, O. Perales-Perez y F. Roman-Velasquez, "Sorption Study of Toluene and Xylene in Aqueous Solutions by Recycled Tires Crumb Rubber," Journal of Hazardous Materials, vol. 185, no. 1, pp. 107-111, 2011. https://doi. org/10.1016/j.jhazmat.2010.09.003

[56] G. San Miguel, G. Fowler y C. Sollars, "Adsorption of Organic Compounds from Solution by Activated Carbons Produced from Waste Tyre Rubber," Separation Science and Technology, vol. 37 , no. 3, pp. 663-676, 2002. https://doi. org/10.1081/ss-120001453

[57] N. Hamadi, S. Swaminathan y X. Chen, "Adsorption of Paraquat Dichloride from Aqueous Solution by Activated Carbon Derived from Used Tires," Journal of Hazardous Materials, vol. 112, no. 1-2, pp. 133-141, 2004. https://doi. org/10.1016/j.jhazmat.2004.04.011

[58] W. Tanthapanichakoon, P. Ariyadejwanich, P. Japthong, K. Nakagawa et al., "Adsorption-desorption Characteristics of Phenol and Reactive Dyes from Aqueous Solution on Mesoporous Activated Carbon Prepared from Waste Tires,"
Water Research, vol. 39, no. 7, pp. 13471353, 2005. https://doi.org/10.1016/j. watres.2004.12.044

[59] F. Calisir, F. Roman, L. Alamo, O. Perales et al., "Removal of Cu (II) from Aqueous Solutions by Recycled Tire Rubber," Desalination, vol. 249, no. 2, pp. 515-518, 2009. https://doi. org/10.1016/j.desal.2008.07.029

[60] N. Hamadi, X. Chen, M. Farid y M. Lu, "Adsorption Kinetics for the Removal of Chromium (VI) from Aqueous Solution by Adsorbents Derived from Used Tyres and Sawdust," Chemical Engineering Journal, vol. 84, no. 2, pp. 95-105, 2001. https://doi.org/10.1016/ s1385-8947(01)00194-2

[61] E. Manchón-Vizuete, A. Macías-García, A. Nadal, C. Fernández-González y V. Gómez-Serrano, "Adsorption of Mercury by Carbonaceous Adsorbents Prepared from Rubber of Tyre Wastes," Journal of Hazardous Materials, vol. 119, no. 1-3, pp. 231-238, 2005. https://doi. org/10.1016/j.jhazmat.2004.12.028

[62] M. Alexandre-Franco, C. Fernández-González, M. Alfaro-Domínguez y V. Gómez-Serrano, "Adsorption of Cadmium on Carbonaceous Adsorbents Developed from Used Tire Rubber," Journal of Environment Management, vol. 92, no. 9, pp. 2193-220, 2011. https://doi.org/10.1016/j.jenvman.2011.04.001

[63] M. Alexandre-Franco, C. Fernández-González, A. Macías-García y V. Gómez-Serrano, "Uptake of Lead by Carbonaceous Adsorbents Developed 
from Tire Rubber," Adsorption, vol. 14, no. 4-5, pp. 591-600, 2008. https://doi. org/10.1007/s10450-008-9115-z

[64] Z. Tang, M. Butkus e Y. Xie, "Enhanced Performance of Crumb Rubber Filtration for Ballast Water Treatment," Chemosphere, vol. 74, no. 10, pp. 13961399, 2009. https://doi.org/10.1016/j. chemosphere.2008.11.048

[65] H. Wang, M. Davidson, Y. Zuo y Z. Ren, "Recycled Tire Crumb Rubber Anodes for Sustainable Power Production in Microbial Fuel Cells," Journal of Power Sources, vol. 196, no. 14, pp. 58635866, 2011. https://doi.org/10.1016/j. jpowsour.2011.01.082

[66] H. Schmid, "Waste recycling methodology specifying widely ranging material types and processing equipment," Alemania. Patent No. DE4444532-A1, Febrero 11, 1999.

[67] P. Kvesic, "Treated rubber and products made therefrum," Estados Unidos. Patent No. US2003215637-A1, 2003.

[68] D. Gu, "Polyurethane and rubber foamed compound material and preparation method thereof," China. Patent No. CN102391639-A. China, Mar. 2012.

[69] A. Cachaço, M. Afonso y M. Pinto, "New Applications for Foam Composites of Polyurethane and Recycled Rubber," Journal of Applied Polymer Science, vol. 129, no.5, pp. 2873-2881, 2013. https://doi.org/10.1002/app.38962

[70] B. Wu y M. Zhou, "Recycling of Waste Tyre Rubber into Oil Absorbent," Waste Management, vol. 29, no. 1, pp. 355-
359, 2009. https://doi.org/10.1016/j. wasman.2008.03.002

[71] American Standard Test Methods [ASTM], "ASTM F726 - Standrad Method of Testing Sorbent Performance of Adsorbents E1," ASTM International, West Conshohocken, PA, 2012. https:// doi.org/10.1520/F0726-12

[72] S. Zhang, Z. Xin, Z. Zhang y J. Kim, "Characterization of the Properties of Thermoplastic Elastomers Containing Waste Rubber Tire Powder," Waste Management, vol. 29, no. 5, pp. 14801485, 2009. https://doi.org/10.1016/j. wasman.2008.10.004

[73] Z. Al-Malki, E. Al-Nasir, M. Khalaf y R. Zidan, "Study the Effect of Recycled Tire Rubber on the Mechanical and Rheological Properties of TPV (HDPE/ Recycled Tire Rubber)," Open Journal of Polymer Chemistry, vol. 3, no. 4, pp. 99-103, 2013. https://doi.org/10.4236/ ojpchem.2013.34017

[74] J.Karger-Kocsis,L. MészárosyT.Bárány, "Ground tyre rubber in thermoplastics, thermosets and rubbers," Journal of Materials Science, vol. 48, no. 1, pp. 1-38, 2013. https://doi.org/10.1007/ s10853-012-6564-2

[75] K. El-Nerm y A. Khalil, "Gamma Irradiation of Treated Waste Rubber Powder and its Composites with Waste Polyethylene," Journal of Vinyl \& Additive Technology, vol. 17, no. 1, pp. 58-63, 2011. https://doi.org/10.1002/vnl.20245

[76] L. Mészáros, T. Bárány, T. Czvikovszky, "EB-promoted recycling of waste tire rubber with polyolefins," Radiation Physics 
and Chemistry, vol. 81, no. 9, pp. 13571360, 2012. https://doi.org/10.1016/j. radphyschem.2011.11.058

[77] P. Lima, S. Magalhães, J. Oliveiray V. Costa, "Eheological Properties of Ground Tyre Rubber Based Thermoplastic Elastomeric Blends," Polymer Testing, vol. 45, pp. 58-67, 2015. https://doi.org/10.1016/j. polymertesting.2015.05.006

[78] V. Panaroni y T. McClellan, "Non-cellular polyurethane composite," Estados Unidos. Patente no. US5254405 A, 19 Oct. 1993.
[79] Y. Duan y S. Stammler, “Aqueous polyurethane dispersion adhesive compositions with improved heat resistance," Canadá. Patent No. CA2197186 A1, 14 Mar. 1996

[80] M. Nehdi y A. Khan, "Cementitious Composites Containing Recycled Tire Rubber: An Overview of Engineering Properties and Potential Applications," Cement, Concrete and Aggregates, vol. 23, no. 1, pp. 3-10, 2001. https://doi.org/10.1520/CCA10519J 